\title{
Facial Mapping: Review of Current Methods
}

\author{
${ }^{1}$ C. Stavrianos, ${ }^{3}$ L. Zouloumis, ${ }^{4} \mathrm{C}$. Papadopoulos, ${ }^{2} \mathrm{~J}$. Emmanouil, \\ ${ }^{5} \mathrm{~N}$. Petalotis and ${ }^{5} \mathrm{P}$. Tsakmalis \\ ${ }^{1}$ Department of Endodontology (Forensic Odontology), \\ ${ }^{2}$ Department of Removable Prosthodontics, School of Dentistry, \\ ${ }^{3}$ Departments of Oral and Maxillofacial Surgery, \\ Aristotle University, Thessaloniki, Greece \\ ${ }^{4}$ Department of Forensic Sciences (Forensic Odontology), \\ University of Glamorgan, Wales, UK \\ ${ }^{5}$ Health Center of Sidirokastro, Greece
}

\begin{abstract}
Forensic Odontology uses a variety of methods in identification of human remains. One of them is done by the use of facial mapping which involves the use of antemortem photographs and comparison with the postmortem skull. The current techniques are: morphological comparison, photoanthropometry or photogrammetry and photographic superimposition. Despite the fact that these techniques are complex and substain a variety of technical restrictions, they are widely approved and applied.
\end{abstract}

Key words: Forensic Odontology, facial mapping, techniques, morphological comparison, photoanthropometry or photogrammetry, photographic superimposition

\section{INTRODUCTION}

Facial images are one of the most valuable pieces of evidence for identification and it is possible in a very high proportion of cases wherein subjects recorded in photographs or on videotapes need to be identified by means of comparison with images of persons of known identity. However, the comparison of facial images is often a difficult task as there may be circumstances that may prevent or render facial recognition (Ventura et al., 2004; Stavrianos, 2009; Davis et al., 2010).

The use of photographs to determine people's identity started in Europe at the end of the 19th century by a French anthropologist, Alphonse Bertillon (1853-1914) who introduced a method of personal identification based on anthropology. Bertillon transformed the French Criminal Justice system when he introduced a series of head and body measurements and descriptions about facial appearance and body type that were assumed to be uniquely identifying, calling the technique Bertillonage (Glassman, 2000; Kleinberg et al., 2007). With the increasing diffusion of surveillance cameras the facial characteristics of the perpetrators are often captured and their images retrieved from surveillance footage are the only evidence linking a suspect to the crime. The identification of suspects has

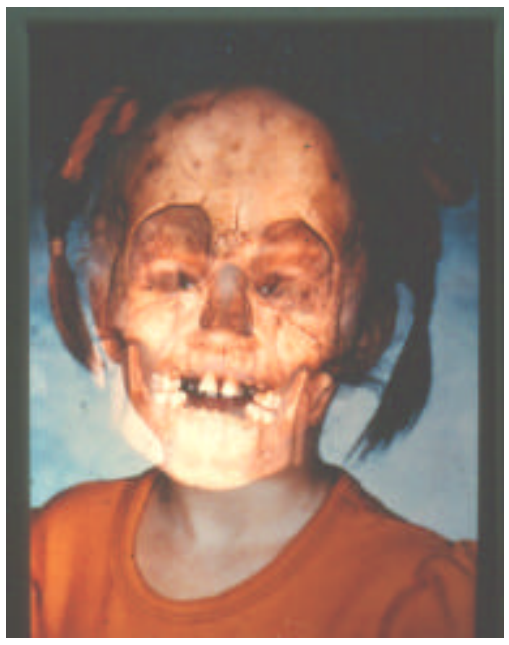

Fig. 1: Superimposition example (Copyright, ABFO, American Board of Forensic Odontology)

been performed either by making comparisons of facial morphology, anthropometry and face-to-face superimposition (Stavrianos, 2009) (Fig. 1). These methods are widely applied, despite the facts that are lacking extensive validation tests and accredited guidelines. Identification by simple morphological classification of traits is obviously insufficient whereas

Corresponding Author: Stavrianos Christos, Department of Endodontology (Forensic Odontology), School of Dentistry, Aristotle University, Thessaloniki, Greece 
the use of facial indices has recently been severely criticized (Lynnerup et al., 2003; Fraser et al., 2003; De Angelis et al., 2009).

\section{FACIAL MAPPING}

Facial mapping is the type of evidence within it experts in facial anatomy and structures provide opinion evidence of identity from examination of evidential facial pictures. This name is perhaps a little misleading and facial comparison is probably more appropriate. However, facial mapping has now become an accepted term for the processes used by imagery analysts who compare faces captured on imagery and comment on their similarity or dissimilarity and this type of evidence is admissible worldwide. The facial mapping requires amalgamation of two principally visual sciences: the forensic photography and morphological anatomy. In forensic practice, there is a frequent demand for comparison of images of perpetrators and suspects (Porter and Doran, 2000; Goos et al., 2006; Oxlee, 2007; Kleinberg et al., 2007; Roelofse et al., 2008; Davis et al., 2010).

\section{TECHNIQUES OF FACIAL MAPPING}

Facial mapping involves the study of the face for forensic purposes using different analytic techniques that are falling into three main categories:

- Making comparisons of facial morphology (morphological comparison)

- Anthropometrical facial comparisons (photoanthropometry or photogrammetry)

- Superimposition (photographic superimposition)

Facial image comparison evidence may comprise one or all of the three facial image comparison techniques to identify potential similarities and differences between two facial images. The dimensions and characteristics of the face on the two photographs are compared to investigate if it belongs to the same person or if it can be excluded from being that person (Roelofse et al., 2008). If the maxilla and mandible are completely or partially edentulous then placing the associated dentures the investigator could assess some important dimensions of the facial structures such as the vertical dimension (nasomental, etc.). These findings are essential in handling with superimposition technique (Stavrianos, 2009).

\section{MORPHOLOGICAL COMPARISON}

It is the detailed morphological comparison of facial features which are graded by shape and size into discrete categories. This morphological investigation is based on the direct examination of the characteristics and on a thorough visual search for specific elements. Once these characteristics of an image depicting an individual have been classified and ordered according to the facial zone, they can be compared with the facial characteristics yielded by the examination of another image (Iscan and Loth, 2000; Ventura et al., 2004).

It is probably the most effective technique with images of poor quality or from different viewpoints as prominent individuating features remain visible. Furthermore, statistical analyses can only be conducted at a nominal level, meaning that discrimination of faces possessing similar characteristics will be problematic (Davis et al., 2010).

\section{PHOTOANTHROPOMETRY}

Photoanthropometry is the science of measurements from precisely defined marker points in various zones of the face or in specific anatomical areas as these appear in photographs. In facial mapping, it is based on quantitative analysis on measurements of the distances and angles between anatomical facial landmarks and the generation of indices based on them. The aim is to compare metrically the proportional relationships of one photograph to another, rather than assess absolute visual differences and similarities as in morphological comparisons (Iscan and Loth, 2000; Davis et al., 2010; Moreton and Morley, 2011).

A key element in the use of photoanthropometry is the formulation of indices based on proportions rather than absolute size. As in all analyses, the landmarks used for indices must be clearly visible and defined if they are not standard sites. The quality and angulation of the image may dictate the use of unusual points that can be clearly defined and located on both images. A number of anthropological soft tissue landmarks are marked on the facial image and the distance between two landmarks is measured. Since, absolute size is not reliable without a scale, proportionality indices must be calculated from these measurements to insure that the values are comparable. Normalized indices are calculated for each measurement, taking each measurement as a percentage of the largest available measurement. An index is created as follows:

$$
\frac{\text { Smaller dimension }}{\text { Larger dimension }} \times 100
$$

It is now accepted that anthropometry indices are not suitably discriminating to positively identify an individual and facial indices comparison are currently only used to test for elimination. If the anthropometry indices of the 
two images are deemed by the experience of the expert to be suitably dissimilar then the two images are determined to not show the same person (Iscan and Loth, 2000; Moreton and Morley, 2011). The techniques that are used in photoanthropometry include:

Measurement: Due to the fact that commonly images under comparison are different in scale, any photoanthropometry comparison must be based on actual measurement after the correct scales have been established. However, this demands detailed knowledge of the technical details of the recording camera which is not generally available to the analyst. Nevertheless, accurate work can be achieved on the original imagery by calculating the ratios between facial landmarks. However, it is important to note that from this evidence alone the analyst could not say that the two were the same because these ratios may be shared by many in the population (Iscan and Loth, 2000; Oxlee, 2007).

Facial alignment: Another technique used is facial alignment. The facial pictures are carefully scaled using the distances between landmarks in the vertical plane and in the horizontal plane. These are then equally carefully aligned and lines drawn through as many landmarks as possible (Iscan and Loth, 2000).

Superimposition: Superimposition of one picture upon another is a further method of testing the reliability of morphological analysis. The most common methodology used is to utilize tracings of one of the images and superimpose it upon the other. As with any research of this kind, great care must be taken with both the scaling and enlargement, particularly when the differences in geometry of the two pictures under comparison are marked. No attempt should be made to rectify the geometry as in this way there is a risk to actually attempt to make one picture fit into another or vice versa (Iscan and Loth, 2000; Oxlee, 2007).

\section{PHOTOGRAPHIC SUPERIMPOSITION}

Photographic superimposition is the method whereby one image is projected over another one in order to determine if facial features and dimensions of the two superimposed photos do match. Superimposition highlights facial similarities, discrepancies as well as the possession or the lack of facial symmetry. The challenging part is judging the quality of fit, identifying inconsistencies and knowing the range of acceptable variability. Superimposition comparisons have developed into a useful tool for identification procedures.
These techniques fall into three general categories: photographic, video and computer aided. All three have been modified several times since their first appearance in the scientific literature and several variations within all three categories are used for identification purposes today. In superimposition cases, there are too many variables that contain some measure of error. These include but are not limited to, the orientation of the faces, size, distortion, features to be used for comparison and the criteria to reach a conclusion of matching or exclusion. The importance of each of these issues for the results and accuracy of superimposition studies has been discussed since the technique was first proposed and the debate continues up today. Thus, superimposition is considered to be an exclusionary tool rather than one of positive identification (Aulsebrook et al., 1995; Iscan and Loth, 2000; Glassman, 2000; Yoshino et al., 2005; Davis et al., 2010).

The superimposition of facial tracings can be made by a forensic expert manually or created by a computer using standard line-detection algorithms. The methods that could be applied are given:

Superimposition of imagery: The facial pictures are superimposed progressively and if there are some differences observed such as the line of the hair but these are termed transitory differences and would not be seen as significant.

Flicker and change detection: To identify differences, analysts use computer systems to alternate between the two pictures. If this is done rapidly, the differences flash (flicker) to the observer. Another method to pinpoint differences is to use software to reveal differences (known as change detection). In this method, the computer will only show differences between the two pictures and can for example, paint these differences in a contrasting color. Differences highlighted in this way can be studied in detail to determine if they are significant or not.

Wiping: The wipes can be horizontal, vertical or even diagonal across the pictures.

Matching: It is sometimes useful for analysts to place the right half of a face against the left half of the face under comparison or vice versa to determine if they match within reason. Once again, care must be taken in drawing conclusions. Human faces are seldom completely symmetrical and anatomical midline is difficult to identify but it is a technique which draws attention to anomalies particularly on poor pictures and may help to detect a meaningful difference between the two persons (Oxlee, 2007). 
Res. J. Med.Sci., 6 (2): 77-82, 2012

\section{COMMON PROBLEMS IN FACIAL MAPPING}

The identification of a person in a forensic situation based on facial features is a complex procedure. The variation in face morphology, the difficulty in providing evidence that would stand up in court and inter-observer repeatability are only some of the problems that makes this a very difficult task. In criminal/civil cases the expert's opinion is usually of a subjective nature with considerable variation between observers and unknown performance rates both of individual experts and in lay people (Kleinberg et al., 2007; Roelofse et al., 2008).

There are several disturbing factors that could have a significant influence in the success of the facial mapping method and reduce the objectivity of the photographic comparisons. These include the unknown focal length of the camera taking the image, the unknown pixel aspect ratio and low image resolution and lens distortion. The photographs are usually also taken under different conditions and different orientation of the face. Other parameters that affect the facial mapping method are change of the location of landmarks due to weight difference and age progression, intended alteration of facial features, differences in facial expressions. The positioning of the head within a $3 \mathrm{D}$ environment is complex and poorly represented in 2D imagery. The ability to physically correct for angular orientations in a $2 \mathrm{D}$ image is severely limited. The rotation of a $2 \mathrm{D}$ image cannot correct for distortion of the images proportions caused by rotation of the head in the medial and transverse planes (Iscan and Loth, 2000; Yoshino et al., 2005; Goos et al., 2006; De Angelis et al., 2009).

\section{DISCUSSION}

Regardless of the facial mapping technique used, only proof of non-identity is possible. Facial comparisons would also be considered better for exclusion than inclusion. A single reliable difference has more weight than multiple similarities. The combination of detailed morphological analysis, anthropometry and superimposition can assist in confirmation or exclusion of identity. Considering the various factors that can influence any facial comparison method it is not surprising that facial comparison has been criticized, particularly as two or more witnesses using similar techniques can come to opposite conclusions (Roelofse et al., 2008; Davis et al., 2010).

Yoshino et al. $(2000,2005)$ dedicated significant research time in facial identification using 3D images. In an attempt to compare images with differing positions developed a $3 \mathrm{D}$ physiognomic range finder that adjusts a $3 \mathrm{D}$ image to match the orientation and size of the $2 \mathrm{D}$ image for facial comparison, incorporating a morphological comparison, an anthropometric analysis and morphometric matching to achieve facial identification. The positions of anatomical landmarks were compared after computer-assisted superimposition of the $3 \mathrm{D}$ models on the 2D images. Their conclusion is that the anthropometric analysis used is helpful in the identification process.

Riccio and Dugelay (2007) proposed an asymmetrical $3 \mathrm{D} / 2 \mathrm{D}$ face recognition technique based on geometric invariants. They have addressed the crucial problem of choosing the control points in case of faces for the $2 \mathrm{D}$ image and 3D model based invariants. They tested their technique in order to assess the robustness of the method with respect to the changes in pose and to the accuracy of locating the control points. The results were encouraging in terms of recognition rate. In the proposed experiments, the localization of the control points has been simulated, picking up the points by hand on the probe and gallery models and then rotating them according to the considered poses.

Among the disadvantages encountered with the superimposition of facial features assessment is the need to have the test images at the same orientation. In practice however, Vanezis and Brierley (1996) found that images which are not at exactly similar viewpoints can still be assessed by direct comparison, provided that the orientation is within tolerable limits. The defining of a tolerable limit is an empirical judgement based on the experience of the assessor, taking into consideration the appearance of facial features from different orientations.

De Angelis et al. (2009) proposed a method which is thought to produce high quality and reliable facial superimposition so that anthropologists or medical examiners may compare facial features and indicate corresponding traits or discrepancies in a court of law. The research concluded that the acquired 3D masks of suspects can be reliably superimposed to the face of offenders and experts can judge the produced images via qualitative and quantitative methods. The disadvantages of this method consist in the high cost of the laser device and the use of 3D modelling software.

The findings from various researches highlight the difficulty in taking facial measurements accurately from photographs. Such measurements, even when defined as ratios with respect to other measurements taken from the same face are highly dependent on uncontrolled conditions such as lighting, expression and pose and it is recognised to be a very serious limitation in facial mapping. Porter and Doran (2000) in their research used the interpupillary distance as a way to ensure that the correct magnification was produced and measurements to determine if the person in three different photographs was 
the same. Their conclusion was that there are certain statistical tests that can help determine the probability of the photographed individuals being the same person. In contrast, experiments performed by Catterick (1992) have shown facial measurements based on facial features to be less successful in facial identification and their application would be limited and photoanthropometry as a facial image comparison technique can at best be inconclusive. In addition, Davis et al. (2010) findings of the photo-anthropometrical analyses using a face landmark identification system illustrate that caution is required if deciding whether two different photographs depict the same person.

Kleinberg et al. (2007) analysed distance and angular measurements derived from a set of four landmarks on a database of 120 male police recruits, concluding that the comparison of video images and photographs using anthropometric proportions from the chosen landmarks, even under nearly ideal conditions, appears to be of limited value in identification cases. The concluded that measurements between facial features alone may be insufficient to distinguish between individuals because it is quite possible to obtain small measurable differences even in the same individual taking into account the numerous variables that can obtain when such images are produced.

Recently, extensive research has been taken place in the field of automatic face dentification using algorithms that appear to be more accurate than humans under optimal conditions at correctly identity matching or discriminating two different faces. Computer algorithms are capable of outperforming people on recognition of frontal face images when large and representative training dataset is available. However, machine based algorithms are still limited in the number of image variations they can generalize across. In facial images captured by different viewpoints and environmental conditions, the accuracy of computer facial recognition systems is worse than human performance. One of the most obvious differences between human and machine is the ability to learn. After a close look at a certain face, people can memorize and recognize that face in many unseen situations such as new pose, lighting and ages. In contrast, face recognition algorithms can only handle simple expression or occlusion changes, lacking the generalization to new complex situations (Davis et al., 2010; Deng et al., 2010).

\section{CONCLUSION}

These methods cannot lead to positive identification in a certain percent of cases but definitely can conclude to disassociation of current remain to a possible identity. Now-a-days, the use of specialized computer programs has broadened the use of facial mapping due to the simplicity of comparison. Further research is needed for establishing accredited guidelines.

\section{REFERENCES}

Aulsebrook, W.A., J.M.Y. Iscan, H. Slabbert and P. Beckerd, 1995. Superimposition and reconstruction in forensic facial identification: A survey. Forensic Sci. Int., 75: 101-120.

Catterick, T., 1992. Facial measurements as an aid to recognition. Forensic Sci. Int., 56: 23-27.

Davis, J.P., T. Valentine and R.E. Davis, 2010. Computer assisted photo-anthropometric analyses of fullface and profile facial images. Forensic Sci. Int., 200: 165-176.

De Angelis, D., R. Sala, A. Cantatore, M. Grandi and C. Cattaneo, 2009. A new computer-assisted technique to aid personal identification. Int. J. Legal Med., 123: 351-356.

Deng, W., J. Hua, J. Guo, W. Cai and D. Feng, 2010. Robust, accurate and efficient face recognition from a single training image: A uniform pursuit approach. Pattern Recognit., 43: 1748-1762.

Fraser, N.L., M. Yoshino, K. Imaizumi, S.A. Blackwell, C.D. Thomas and J.G.A. Clement, 2003. Japanese computer-assisted facial identification system successfully identifies non-Japanese faces. Forensic Sci. Int., 135: 122-128.

Glassman, D.M., 2000. Methods of Superimposition. In: In: Forensic Art and illustration, Taylor, K.T. (Ed.)., CRC Press, 477-497.

Goos, M.I.M., I.B. Alberink and A.C.C. Ruifrok, 2006. $2 \mathrm{D} / 3 \mathrm{D}$ image (facial) comparison using camera matching. Forensic Sci. Int., 163: 10-17.

Iscan, M.Y. and S.R. Loth, 2000. Photo Image Identification. In: In: Encyclopaedia of Forensic Science, Siegel, J., G. Knupfer and P. Saukko (Eds.)., Academic Press, pp: 795-807.

Kleinberg, K.F., P. Vanezisand and A.M. Burton, 2007. Failure of anthropometry as a facial identification technique using high-quality photographs. J. Forensic Sci., 52: 779-783.

Lynnerup, N., M. Andersen and H.P. Lauritsen, 2003. Facial image identification using Photomodeler. Legal Med., 5: 156-160.

Moreton, R. and J. Morley, 2011. Investigation into the use of photoanthropometry in facial image comparison. Forensic Sci. Int., 212: 231-237. 
Oxlee, G., 2007. Facial Recognition and Imagery Analysis. In: In: Forensic human identification, Thompson, $\mathrm{T}$. and S. Black (Eds.)., CRC Press, Taylor and Francis Group, pp: 257-270.

Porter, G. and G. Doran, 2000. An anatomical and photographic technique for forensic facial identification. Forensic Sci. Int., 114: 97-105.

Riccio, D. and J.L. Dugelay, 2007. Geometric invariants for 2D/3D face recognition. Pattern Recognit. Lett., 28: 1907-1914.

Roelofse, M.M., M. Steyn and P.J. Becker, 2008. Photo identification: Facial metrical and morphological features in South African males. Forensic Sci. Int., 177: 168-175.

Stavrianos, C., 2009. Forensic Dentistry. 1stEdn., Vol. 1-2, University Studio Press, Thessaloniki, pp: 45-98.
Vanezis, P. and C. Brierley, 1996. Facial image comparison of crime suspects using video superimposition. Sci. Justice, 36: 27-34.

Ventura, F., A. Zacheo, A. Ventura and A. Pala, 2004. Computerised anthropomorphometric analysis of images: Case report. Forensic Sci. Int., 146: 211-213.

Yoshino, M., H. Matsuda, S. Kubota, K. Imaizumi and S. Miyasaka, 2000. Computer-assisted facial image identification system using a 3-D physiognomic range finder. Forensic Sci. Int., 109: 225-237.

Yoshino, M., M. Taniguchi, K. Imaizumi, S. Miyasaka and T. Tanijiri et al., 2005. A new retrieval system for a database of 3D facial images. Forensic Sci. Int., 148: $113-120$. 